Acta Crystallographica Section E

Structure Reports

Online

ISSN 1600-5368

\section{Bis[4-(2-hydroxyethylamino)phenyl] sulfone}

\section{Guo-Feng Chen, ${ }^{\mathrm{a}, \mathrm{b}}$ Guo-Chun $\mathrm{Ma}^{,}{ }^{\mathrm{a}}$ Jing $\mathrm{Hu}^{\mathrm{a}}$ and Wen-Qin Zhang $^{\text {a* }}$}

a'Department of Chemistry, Tianjin University, Tianjin 300072, People's Republic of China, and ${ }^{\mathbf{b}}$ Department of Chemistry, College of Chemistry and Environmental Science, Hebei University, Baoding 071002, People's Republic of China

Correspondence e-mail: chenguofeng@mail.hbu.edu.cn

Received 12 November 2007; accepted 27 November 2007

Key indicators: single-crystal X-ray study; $T=294 \mathrm{~K}$; mean $\sigma(\mathrm{C}-\mathrm{C})=0.003 \AA$; $R$ factor $=0.037 ; w R$ factor $=0.110 ;$ data-to-parameter ratio $=12.6$.

The title compound, $\mathrm{C}_{16} \mathrm{H}_{20} \mathrm{~N}_{2} \mathrm{O}_{4} \mathrm{~S}$, exhibits a V-shape structure with a dihedral angle of $77.5(11)^{\circ}$ formed by the two benzenel rings. The molecular packing is stabilized by intramolecular and intermolecular hydrogen bonds as well as $\pi-\pi[3.738(3) \AA]$ and $\mathrm{C}-\mathrm{H} \cdots \pi$ interactions.

\section{Related literature}

For related literature, see: Shahsafi et al. (1987).<smiles>O=S(=O)(c1ccc(NCCO)cc1)c1ccc(NCCO)cc1</smiles>

\section{Experimental}

Crystal data

$\mathrm{C}_{16} \mathrm{H}_{20} \mathrm{~N}_{2} \mathrm{O}_{4} \mathrm{~S}$

$M_{r}=336.40$

Monoclinic, $C 2 / c$

$a=25.643$ (17)
$Z=8$

Mo $K \alpha$ radiation

$\mu=0.23 \mathrm{~mm}^{-1}$

Data collection

Bruker SMART CCD area-detector diffractometer

Absorption correction: multi-scan (SADABS; Sheldrick, 1996)

$T_{\min }=0.953, T_{\max }=0.966$

Refinement

$R\left[F^{2}>2 \sigma\left(F^{2}\right)\right]=0.037$

$w R\left(F^{2}\right)=0.110$

$S=1.03$

2742 reflections

218 parameters

2 restraints
$T=294$ (2) K

$0.20 \times 0.18 \times 0.16 \mathrm{~mm}$

7793 measured reflections 2742 independent reflections 1982 reflections with $I>2 \sigma$ $R_{\text {int }}=0.033$

$\mathrm{H}$ atoms treated by a mixture of independent and constrained refinement

$\Delta \rho_{\max }=0.22{\mathrm{e} \AA^{-3}}^{-3}$

$\Delta \rho_{\min }=-0.35{\mathrm{e} \AA^{-3}}^{-3}$

Table 1

Hydrogen-bond geometry $\left(\AA,^{\circ}\right)$.

\begin{tabular}{lllll}
\hline$D-\mathrm{H} \cdots A$ & $D-\mathrm{H}$ & $\mathrm{H} \cdots A$ & $D \cdots A$ & $D-\mathrm{H} \cdots A$ \\
\hline $\mathrm{C} 14-\mathrm{H} 14 \cdots \mathrm{O} 3$ & 0.93 & 2.59 & $2.919(3)$ & 101 \\
$\mathrm{C} 10-\mathrm{H} 10 \cdots \mathrm{O} 2$ & 0.93 & 2.57 & $2.915(3)$ & 102 \\
$\mathrm{C} 7-\mathrm{H} 7 \cdots \mathrm{O} 3$ & 0.93 & 2.47 & $2.854(3)$ & 105 \\
$\mathrm{O} 1-\mathrm{H} 1 \cdots \mathrm{O} 3{ }^{\mathrm{i}}$ & 0.82 & 1.87 & $2.683(3)$ & 175 \\
$\mathrm{O} 1-\mathrm{H} 1 \cdots \mathrm{S} 1^{\mathrm{i}}$ & 0.82 & 2.88 & $3.638(2)$ & 154 \\
$\mathrm{O} 4-\mathrm{H} 4 \cdots \mathrm{O} 2^{\text {ii }}$ & 0.82 & 2.13 & $2.945(3)$ & 177 \\
$\mathrm{~N} 1-\mathrm{H} 1 C \cdots \mathrm{O} 4^{\text {iii }}$ & $0.892(10)$ & $2.185(11)$ & $3.066(3)$ & $169(2)$ \\
$\mathrm{N} 2-\mathrm{H} 2 C \cdots \mathrm{O} 1^{\text {iv }}$ & $0.895(10)$ & $2.027(11)$ & $2.917(3)$ & $172(2)$ \\
$\mathrm{C} 10-\mathrm{H} 10 \cdots C g 2$ & 0.93 & 2.97 & $3.762(4)$ & 144 \\
\hline Symmetry codes: $(\mathrm{i})-x, y+1,-z+\frac{1}{2} ;\left(\right.$ ii) $-x+\frac{1}{2}, y-\frac{1}{2},-z+\frac{1}{2} ;\left(\right.$ iii) $x-\frac{1}{2}, y+\frac{1}{2}, z ;(\mathrm{iv})$ \\
$x+\frac{1}{2}, y-\frac{1}{2}, z ;(\mathrm{v})-x+\frac{1}{2}, y+\frac{1}{2},-z+\frac{1}{2}$.
\end{tabular}

Data collection: SMART (Bruker, 1997); cell refinement: SAINT (Bruker, 1997); data reduction: $S A I N T$; $\operatorname{program}(\mathrm{s})$ used to solve structure: SHELXTL (Bruker, 2001); program(s) used to refine structure: SHELXTL; molecular graphics: SHELXTL; software used to prepare material for publication: $S H E L X T L$.

Supplementary data and figures for this paper are available from the IUCr electronic archives (Reference: BX2122).

\title{
References
}

Bruker (1997). SMART and SAINT. Bruker AXS Inc., Madison, Wisconsin, USA.

Bruker (2001). SHELXTL. Version 6.12. Bruker AXS Inc., Madison, Wisconsin, USA.

Shahsafi, M. A., Meshkatalsadat, M. H. \& Parekh, H. (1987). Indian J. Chem. 26B, 803-807.

Sheldrick, G. M. (1996). SADABS. University of Göttingen, Germany. 


\section{supporting information}

Acta Cryst. (2008). E64, o174 [https://doi.org/10.1107/S1600536807063805]

\section{Bis[4-(2-hydroxyethylamino)phenyl] sulfone}

\section{Guo-Feng Chen, Guo-Chun Ma, Jing Hu and Wen-Qin Zhang}

\section{S1. Comment}

The derivatives of diphenyl sulphone are used as precursors in the organic synthesis. Several derivatives of aminosulphones have been shown to possess strong tuberculostatic, antileprotic and anticonvulsant activities (Shahsafi, et al., 1987). The crystal structure determination of the title compound, (I), was carried out in order to elucidate its molecular conformation.

The V-shape structure of the molecule is supported by the two phenyl rings with a dihedral angle of $77.5(11)^{\circ}$.

The molecular packing is stabilized by intramolecular and intermolecular hydrogen bonds (Table 1) as well as weak $\pi-\pi$ and $\mathrm{C}-\mathrm{H} . . \pi$ interactions.

\section{S2. Experimental}

The title compound, (I), was synthesized by the reaction of 4,4'-dichlorodiphenyl sulfone (5.74 g, 0.02 mol) with 2aminoethanol $(9.76 \mathrm{~g}, 0.16 \mathrm{~mol})$. The mixture was refluxed for $6 \mathrm{~h}$ and cooled to room temperature. After dilution with water, it was filtered off, washed thoroughly with water, and recrystallized from dimethylformamide and water $(4: 1 \mathrm{v} / \mathrm{v})$ to give the product as fine white needles $(5.5 \mathrm{~g}$, yield $81.8 \%)$. The pure product $(0.1 \mathrm{~g})$ was dissolved in $15 \mathrm{ml}$ dimethylformamide and water $(4: 1 v / v)$. Single crystals were obtained from this solution by slow evaporation over a period of 7 days at room temperature (m.p. 464-466 K).

\section{S3. Refinement}

The $\mathrm{H}$ atom involved in the hydrogen bonds was found in difference Fourier maps. All other $\mathrm{H}$ atoms were positioned geometrically and refined using a during refinement, fix at $\mathrm{O}-\mathrm{H}$ distances of $0.82 \AA$ and its $U_{\text {iso }}$ value was set at $1.2 U_{\text {eq }}$ (O). $\mathrm{H}$ atoms bonded to $\mathrm{C}$ atoms were included in the refinement in the riding model approximation, with $\mathrm{C}-\mathrm{H}=0.93 \AA$. and $U_{\text {iso }}(\mathrm{H})=1.2 U_{\text {eq }}(\mathrm{C}$ atom $)$.

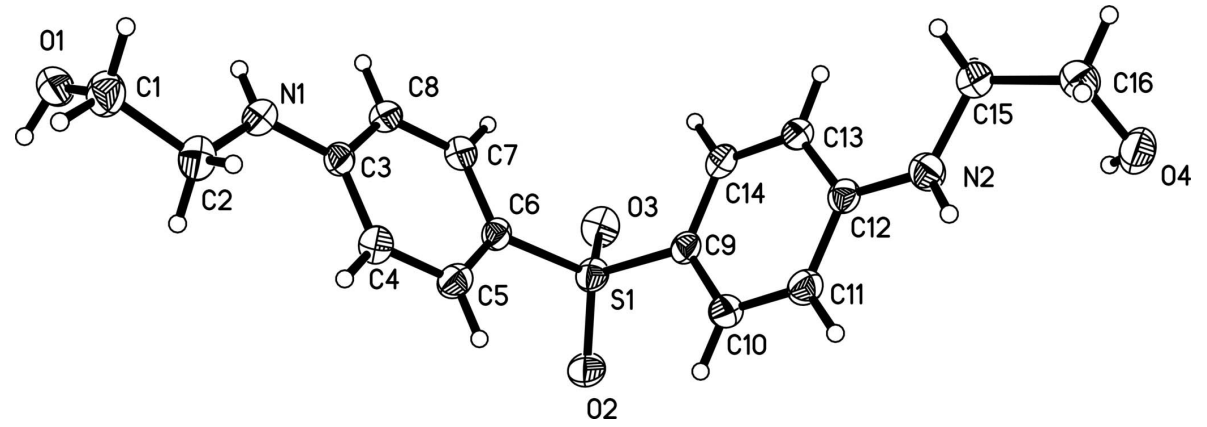

Figure 1

A view of the structure of (I), showing the atom-numbering Scheme; displacement ellipsoids were drawn at the $30 \%$ probability level. 
Bis[4-(2-hydroxyethylamino)phenyl] sulfone

Crystal data

$\mathrm{C}_{16} \mathrm{H}_{20} \mathrm{~N}_{2} \mathrm{O}_{4} \mathrm{~S}$

$M_{r}=336.40$

Monoclinic, $C 2 / c$

Hall symbol: -C $2 \mathrm{yc}$

$a=25.643$ (17) $\AA$

$b=8.118(6) \AA$

$c=15.340(11) \AA$

$\beta=102.989(12)^{\circ}$

$V=3112(4) \AA^{3}$

$Z=8$

\section{Data collection}

Bruker SMART CCD area-detector diffractometer

Radiation source: fine-focus sealed tube Graphite monochromator $\varphi$ and $\omega$ scans

Absorption correction: multi-scan (SADABS; Sheldrick, 1996)

$T_{\min }=0.953, T_{\max }=0.966$

\section{Refinement}

Refinement on $F^{2}$

Least-squares matrix: full

$R\left[F^{2}>2 \sigma\left(F^{2}\right)\right]=0.037$

$w R\left(F^{2}\right)=0.110$

$S=1.03$

2742 reflections

218 parameters

2 restraints

Primary atom site location: structure-invariant direct methods
$F(000)=1424$

$D_{\mathrm{x}}=1.436 \mathrm{Mg} \mathrm{m}^{-3}$

Melting point: $465(1) \mathrm{K}$

Mo $K \alpha$ radiation, $\lambda=0.71073 \AA$

Cell parameters from 2679 reflections

$\theta=2.6-26.4^{\circ}$

$\mu=0.23 \mathrm{~mm}^{-1}$

$T=294 \mathrm{~K}$

Needle, colorless

$0.20 \times 0.18 \times 0.16 \mathrm{~mm}$

7793 measured reflections

2742 independent reflections

1982 reflections with $I>2 \sigma$

$R_{\text {int }}=0.033$

$\theta_{\text {max }}=25.0^{\circ}, \theta_{\min }=1.6^{\circ}$

$h=-26 \rightarrow 30$

$k=-9 \rightarrow 9$

$l=-15 \rightarrow 18$

Secondary atom site location: difference Fourier map

Hydrogen site location: inferred from neighbouring sites

$\mathrm{H}$ atoms treated by a mixture of independent and constrained refinement

$w=1 /\left[\sigma^{2}\left(F_{\mathrm{o}}^{2}\right)+(0.055 P)^{2}+1.5895 P\right]$ where $P=\left(F_{\mathrm{o}}{ }^{2}+2 F_{\mathrm{c}}{ }^{2}\right) / 3$

$(\Delta / \sigma)_{\max }=0.005$

$\Delta \rho_{\max }=0.22 \mathrm{e} \AA^{-3}$

$\Delta \rho_{\min }=-0.35$ e $\AA^{-3}$

\section{Special details}

Geometry. All e.s.d.'s (except the e.s.d. in the dihedral angle between two 1.s. planes) are estimated using the full covariance matrix. The cell e.s.d.'s are taken into account individually in the estimation of e.s.d.'s in distances, angles and torsion angles; correlations between e.s.d.'s in cell parameters are only used when they are defined by crystal symmetry. An approximate (isotropic) treatment of cell e.s.d.'s is used for estimating e.s.d.'s involving 1.s. planes.

Refinement. Refinement of $F^{2}$ against ALL reflections. The weighted $R$-factor $w R$ and goodness of fit $S$ are based on $F^{2}$, conventional $R$-factors $R$ are based on $F$, with $F$ set to zero for negative $F^{2}$. The threshold expression of $F^{2}>\sigma\left(F^{2}\right)$ is used only for calculating $R$-factors (gt) etc. and is not relevant to the choice of reflections for refinement. $R$-factors based on $F^{2}$ are statistically about twice as large as those based on $F$, and $R$ - factors based on ALL data will be even larger.

Fractional atomic coordinates and isotropic or equivalent isotropic displacement parameters $\left(\AA^{2}\right)$

\begin{tabular}{lllll}
\hline & $x$ & $y$ & $z$ & $U_{\text {iso }} * / U_{\text {eq }}$ \\
\hline S1 & $0.13804(2)$ & $0.13061(7)$ & $0.27577(4)$ & $0.0437(2)$ \\
O1 & $-0.11464(7)$ & $0.7991(2)$ & $0.07807(12)$ & $0.0614(5)$ \\
H1 & -0.1153 & 0.8496 & 0.1241 & $0.092 *$
\end{tabular}




\begin{tabular}{|c|c|c|c|c|}
\hline $\mathrm{O} 2$ & $0.16477(7)$ & $0.1933(2)$ & $0.36085(10)$ & $0.0575(5)$ \\
\hline $\mathrm{O} 3$ & $0.11181(7)$ & $-0.0265(2)$ & $0.27233(11)$ & $0.0551(5)$ \\
\hline $\mathrm{O} 4$ & $0.37876(7)$ & $-0.1123(3)$ & $0.00979(13)$ & $0.0656(5)$ \\
\hline $\mathrm{H} 4$ & 0.3659 & -0.1633 & 0.0460 & $0.098 *$ \\
\hline N1 & $-0.02318(8)$ & $0.5964(3)$ & $0.09390(13)$ & $0.0496(5)$ \\
\hline N2 & $0.29293(8)$ & $0.0802(3)$ & $0.05074(14)$ & $0.0479(5)$ \\
\hline $\mathrm{C} 1$ & $-0.06999(11)$ & $0.8469(3)$ & $0.04713(18)$ & $0.0605(7)$ \\
\hline $\mathrm{H} 1 \mathrm{~A}$ & -0.0667 & 0.9659 & 0.0504 & $0.073^{*}$ \\
\hline H1B & -0.0749 & 0.8150 & -0.0151 & $0.073^{*}$ \\
\hline $\mathrm{C} 2$ & -0.01965 (10) & $0.7710(3)$ & 0.09995 (18) & $0.0541(7)$ \\
\hline $\mathrm{H} 2 \mathrm{~A}$ & 0.0106 & 0.8089 & 0.0771 & $0.065^{*}$ \\
\hline $\mathrm{H} 2 \mathrm{~B}$ & -0.0140 & 0.8045 & 0.1621 & $0.065^{*}$ \\
\hline $\mathrm{C} 3$ & $0.01441(8)$ & $0.4931(3)$ & $0.13841(14)$ & $0.0395(5)$ \\
\hline $\mathrm{C} 4$ & $0.06235(9)$ & $0.5465(3)$ & $0.19253(16)$ & $0.0455(6)$ \\
\hline $\mathrm{H} 4 \mathrm{~A}$ & 0.0692 & 0.6588 & 0.1996 & $0.055^{*}$ \\
\hline $\mathrm{C} 5$ & $0.09948(9)$ & $0.4366(3)$ & $0.23534(16)$ & $0.0452(6)$ \\
\hline H5 & 0.1314 & 0.4743 & 0.2712 & $0.054 *$ \\
\hline C6 & $0.09002(8)$ & $0.2708(3)$ & $0.22587(14)$ & $0.0370(5)$ \\
\hline $\mathrm{C} 7$ & $0.04241(9)$ & $0.2161(3)$ & $0.17295(15)$ & $0.0436(6)$ \\
\hline $\mathrm{H} 7$ & 0.0358 & 0.1036 & 0.1664 & $0.052 *$ \\
\hline $\mathrm{C} 8$ & $0.00543(9)$ & $0.3235(3)$ & $0.13085(15)$ & $0.0449(6)$ \\
\hline $\mathrm{H} 8$ & -0.0267 & 0.2844 & 0.0961 & $0.054 *$ \\
\hline C9 & $0.18453(8)$ & $0.1155(3)$ & $0.21042(14)$ & $0.0380(5)$ \\
\hline $\mathrm{C} 10$ & $0.23154(9)$ & $0.2043(3)$ & $0.23028(15)$ & $0.0441(6)$ \\
\hline H10 & 0.2389 & 0.2729 & 0.2801 & $0.053^{*}$ \\
\hline $\mathrm{C} 11$ & $0.26711(9)$ & $0.1918(3)$ & $0.17704(16)$ & $0.0450(6)$ \\
\hline H11 & 0.2989 & 0.2510 & 0.1914 & $0.054 *$ \\
\hline $\mathrm{C} 12$ & $0.25677(9)$ & 0.0920 & $0.10144(14)$ & $0.0378(5)$ \\
\hline $\mathrm{C} 13$ & $0.20852(8)$ & 0.0063 & $0.08150(15)$ & $0.0407(5)$ \\
\hline H13 & 0.2002 & -0.0593 & 0.0305 & $0.049^{*}$ \\
\hline $\mathrm{C} 14$ & $0.17353(9)$ & $0.0173(3)$ & $0.13568(15)$ & $0.0424(6)$ \\
\hline H14 & 0.1418 & -0.0423 & 0.1221 & $0.051^{*}$ \\
\hline $\mathrm{C} 15$ & $0.28658(9)$ & $-0.0244(3)$ & $-0.02587(15)$ & $0.0465(6)$ \\
\hline $\mathrm{H} 15 \mathrm{~A}$ & 0.2595 & 0.0214 & -0.0743 & $0.056^{*}$ \\
\hline H15B & 0.2745 & -0.1321 & -0.0115 & $0.056^{*}$ \\
\hline $\mathrm{C} 16$ & $0.33752(10)$ & $-0.0418(4)$ & $-0.05525(17)$ & $0.0554(7)$ \\
\hline H16A & 0.3313 & -0.1096 & -0.1087 & $0.066^{*}$ \\
\hline H16B & 0.3489 & 0.0661 & -0.0709 & $0.066^{*}$ \\
\hline $\mathrm{H} 1 \mathrm{C}$ & $-0.0542(6)$ & $0.548(3)$ & $0.0693(15)$ & $0.053(7)^{*}$ \\
\hline $\mathrm{H} 2 \mathrm{C}$ & $0.3197(7)$ & $0.153(2)$ & $0.0623(15)$ & $0.049(7)^{*}$ \\
\hline
\end{tabular}

Atomic displacement parameters $\left(\AA^{2}\right)$

\begin{tabular}{lllllll}
\hline & $U^{11}$ & $U^{22}$ & $U^{33}$ & $U^{12}$ & $U^{13}$ & $U^{23}$ \\
\hline S1 & $0.0429(3)$ & $0.0529(4)$ & $0.0352(3)$ & $0.0069(3)$ & $0.0084(2)$ & $0.0087(3)$ \\
O1 & $0.0496(10)$ & $0.0687(12)$ & $0.0647(12)$ & $0.0064(9)$ & $0.0100(9)$ & $-0.0226(10)$ \\
O2 & $0.0570(10)$ & $0.0800(13)$ & $0.0318(9)$ & $0.0134(10)$ & $0.0023(8)$ & $0.0028(8)$ \\
O3 & $0.0590(10)$ & $0.0523(10)$ & $0.0570(11)$ & $-0.0009(9)$ & $0.0190(9)$ & $0.0179(8)$
\end{tabular}


supporting information

\begin{tabular}{|c|c|c|c|c|c|c|}
\hline $\mathrm{O} 4$ & $0.0422(10)$ & $0.0902(15)$ & $0.0653(12)$ & $0.0090(10)$ & $0.0144(9)$ & $0.0052(11)$ \\
\hline N1 & $0.0434(12)$ & $0.0531(13)$ & $0.0481(12)$ & $0.0080(11)$ & $0.0016(10)$ & $-0.0008(10)$ \\
\hline $\mathrm{N} 2$ & $0.0427(11)$ & $0.0510(12)$ & $0.0521(12)$ & $-0.0059(10)$ & $0.0150(10)$ & $-0.0089(10)$ \\
\hline $\mathrm{C} 1$ & $0.0681(18)$ & $0.0621(17)$ & $0.0520(16)$ & $0.0196(15)$ & $0.0147(14)$ & $0.0100(14)$ \\
\hline $\mathrm{C} 2$ & $0.0517(15)$ & $0.0544(16)$ & $0.0578(16)$ & $0.0082(13)$ & $0.0157(12)$ & $0.0068(13)$ \\
\hline $\mathrm{C} 3$ & $0.0367(12)$ & $0.0505(14)$ & $0.0329(12)$ & $0.0077(11)$ & $0.0113(10)$ & $0.0001(10)$ \\
\hline $\mathrm{C} 4$ & $0.0424(13)$ & $0.0445(14)$ & $0.0500(14)$ & $0.0024(11)$ & $0.0111(11)$ & $0.0014(11)$ \\
\hline $\mathrm{C} 5$ & $0.0362(12)$ & $0.0540(15)$ & $0.0442(13)$ & $-0.0014(11)$ & $0.0064(10)$ & $0.0011(11)$ \\
\hline C6 & $0.0331(11)$ & $0.0470(13)$ & $0.0325(11)$ & $0.0049(10)$ & $0.0103(9)$ & $0.0036(10)$ \\
\hline $\mathrm{C} 7$ & $0.0421(13)$ & $0.0457(14)$ & $0.0436(13)$ & $0.0019(12)$ & $0.0110(11)$ & $-0.0027(11)$ \\
\hline $\mathrm{C} 8$ & $0.0359(12)$ & $0.0553(15)$ & $0.0413(13)$ & $0.0018(11)$ & $0.0043(10)$ & $-0.0075(11)$ \\
\hline C9 & $0.0347(11)$ & $0.0411(12)$ & $0.0361(12)$ & $0.0082(10)$ & $0.0034(9)$ & $0.0048(10)$ \\
\hline $\mathrm{C} 10$ & $0.0430(13)$ & $0.0439(14)$ & $0.0426(13)$ & $0.0038(11)$ & $0.0040(10)$ & $-0.0037(11)$ \\
\hline $\mathrm{C} 11$ & $0.0371(12)$ & $0.0441(13)$ & $0.0518(15)$ & $-0.0028(11)$ & $0.0060(11)$ & $-0.0040(12)$ \\
\hline $\mathrm{C} 12$ & $0.0361(11)$ & $0.0375(12)$ & $0.0386(12)$ & $0.0050(10)$ & $0.0059(10)$ & $0.0038(10)$ \\
\hline C13 & $0.0358(12)$ & $0.0420(13)$ & $0.0414(13)$ & $0.0036(10)$ & $0.0024(10)$ & $-0.0050(11)$ \\
\hline $\mathrm{C} 14$ & $0.0316(11)$ & $0.0459(13)$ & $0.0472(13)$ & $0.0030(10)$ & $0.0034(10)$ & $0.0023(11)$ \\
\hline $\mathrm{C} 15$ & $0.0424(13)$ & $0.0533(15)$ & $0.0428(13)$ & $0.0017(11)$ & $0.0073(11)$ & $-0.0014(11)$ \\
\hline C16 & $0.0544(15)$ & $0.0697(18)$ & $0.0444(14)$ & $-0.0022(14)$ & $0.0158(12)$ & $-0.0033(13)$ \\
\hline
\end{tabular}

Geometric parameters $\left(\AA,{ }^{\circ}\right)$

\begin{tabular}{llll}
\hline $\mathrm{S} 1-\mathrm{O} 2$ & $1.4249(18)$ & $\mathrm{C} 4-\mathrm{H} 4 \mathrm{~A}$ & 0.9300 \\
$\mathrm{~S} 1-\mathrm{O} 3$ & $1.4371(19)$ & $\mathrm{C} 5-\mathrm{C} 6$ & $1.370(3)$ \\
$\mathrm{S} 1-\mathrm{C} 6$ & $1.724(2)$ & $\mathrm{C} 5-\mathrm{H} 5$ & 0.9300 \\
$\mathrm{~S} 1-\mathrm{C} 9$ & $1.726(2)$ & $\mathrm{C} 6-\mathrm{C} 7$ & $1.379(3)$ \\
$\mathrm{O} 1-\mathrm{C} 1$ & $1.390(3)$ & $\mathrm{C} 7-\mathrm{C} 8$ & $1.342(3)$ \\
$\mathrm{O} 1-\mathrm{H} 1$ & 0.8200 & $\mathrm{C} 7-\mathrm{H} 7$ & 0.9300 \\
$\mathrm{O} 4-\mathrm{C} 16$ & $1.402(3)$ & $\mathrm{C} 8-\mathrm{H} 8$ & 0.9300 \\
$\mathrm{O} 4-\mathrm{H} 4$ & 0.8200 & $\mathrm{C} 9-\mathrm{C} 14$ & $1.373(3)$ \\
$\mathrm{N} 1-\mathrm{C} 3$ & $1.343(3)$ & $\mathrm{C} 9-\mathrm{C} 10$ & $1.379(3)$ \\
$\mathrm{N} 1-\mathrm{C} 2$ & $1.422(3)$ & $\mathrm{C} 10-\mathrm{C} 11$ & $1.358(3)$ \\
$\mathrm{N} 1-\mathrm{H} 1 \mathrm{C}$ & $0.892(10)$ & $\mathrm{C} 10-\mathrm{H} 10$ & 0.9300 \\
$\mathrm{~N} 2-\mathrm{C} 12$ & $1.341(3)$ & $\mathrm{C} 11-\mathrm{C} 12$ & $1.390(3)$ \\
$\mathrm{N} 2-\mathrm{C} 15$ & $1.429(3)$ & $\mathrm{C} 11-\mathrm{H} 11$ & 0.9300 \\
$\mathrm{~N} 2-\mathrm{H} 2 \mathrm{C}$ & $0.895(10)$ & $\mathrm{C} 12-\mathrm{C} 13$ & $1.393(3)$ \\
$\mathrm{C} 1-\mathrm{C} 2$ & $1.494(4)$ & $\mathrm{C} 13-\mathrm{C} 14$ & $1.356(3)$ \\
$\mathrm{C} 1-\mathrm{H} 1 \mathrm{~A}$ & 0.9700 & $\mathrm{C} 13-\mathrm{H} 13$ & 0.9300 \\
$\mathrm{C} 1-\mathrm{H} 1 \mathrm{~B}$ & 0.9700 & $\mathrm{C} 14-\mathrm{H} 14$ & 0.9300 \\
$\mathrm{C} 2-\mathrm{H} 2 \mathrm{~A}$ & 0.9700 & $\mathrm{C} 15-\mathrm{C} 16$ & $1.481(3)$ \\
$\mathrm{C} 2-\mathrm{H} 2 \mathrm{~B}$ & 0.9700 & $\mathrm{C} 15-\mathrm{H} 15 \mathrm{~A}$ & 0.9700 \\
$\mathrm{C} 3-\mathrm{C} 4$ & $1.389(3)$ & $\mathrm{C} 15-\mathrm{H} 15 \mathrm{~B}$ & 0.9700 \\
$\mathrm{C} 3-\mathrm{C} 8$ & $1.396(3)$ & $\mathrm{C} 16-\mathrm{H} 16 \mathrm{~A}$ & 0.9700 \\
$\mathrm{C} 4-\mathrm{C} 5$ & $1.361(3)$ & $\mathrm{C} 16-\mathrm{H} 16 \mathrm{~B}$ & 0.9700 \\
& & & $119.89(18)$ \\
$\mathrm{O} 2-\mathrm{S} 1-\mathrm{O} 3$ & $118.39(11)$ & $\mathrm{C} 7-\mathrm{C} 6-\mathrm{S} 1$ & $120.7(2)$ \\
$\mathrm{O} 2-\mathrm{S} 1-\mathrm{C} 6$ & $108.63(11)$ & $\mathrm{C} 8-\mathrm{C} 7-\mathrm{C} 6$ & 119.7 \\
$\mathrm{O} 3-\mathrm{S} 1-\mathrm{C} 6$ & $106.71(11)$ & $\mathrm{C} 8-\mathrm{C} 7-\mathrm{H} 7$ &
\end{tabular}




\begin{tabular}{|c|c|c|c|}
\hline $\mathrm{O} 2-\mathrm{S} 1-\mathrm{C} 9$ & $107.72(11)$ & $\mathrm{C} 6-\mathrm{C} 7-\mathrm{H} 7$ & 119.7 \\
\hline $\mathrm{O} 3-\mathrm{S} 1-\mathrm{C} 9$ & $107.12(11)$ & $\mathrm{C} 7-\mathrm{C} 8-\mathrm{C} 3$ & $121.0(2)$ \\
\hline $\mathrm{C} 6-\mathrm{S} 1-\mathrm{C} 9$ & $107.85(11)$ & $\mathrm{C} 7-\mathrm{C} 8-\mathrm{H} 8$ & 119.5 \\
\hline $\mathrm{C} 1-\mathrm{O} 1-\mathrm{H} 1$ & 109.5 & $\mathrm{C} 3-\mathrm{C} 8-\mathrm{H} 8$ & 119.5 \\
\hline $\mathrm{C} 16-\mathrm{O} 4-\mathrm{H} 4$ & 109.5 & $\mathrm{C} 14-\mathrm{C} 9-\mathrm{C} 10$ & $119.6(2)$ \\
\hline $\mathrm{C} 3-\mathrm{N} 1-\mathrm{C} 2$ & $124.2(2)$ & $\mathrm{C} 14-\mathrm{C} 9-\mathrm{S} 1$ & $119.16(18)$ \\
\hline $\mathrm{C} 3-\mathrm{N} 1-\mathrm{H} 1 \mathrm{C}$ & $114.2(16)$ & $\mathrm{C} 10-\mathrm{C} 9-\mathrm{S} 1$ & $121.22(18)$ \\
\hline $\mathrm{C} 2-\mathrm{N} 1-\mathrm{H} 1 \mathrm{C}$ & $120.3(16)$ & $\mathrm{C} 11-\mathrm{C} 10-\mathrm{C} 9$ & $120.0(2)$ \\
\hline $\mathrm{C} 12-\mathrm{N} 2-\mathrm{C} 15$ & $123.6(2)$ & $\mathrm{C} 11-\mathrm{C} 10-\mathrm{H} 10$ & 120.0 \\
\hline $\mathrm{C} 12-\mathrm{N} 2-\mathrm{H} 2 \mathrm{C}$ & $115.9(15)$ & $\mathrm{C} 9-\mathrm{C} 10-\mathrm{H} 10$ & 120.0 \\
\hline $\mathrm{C} 15-\mathrm{N} 2-\mathrm{H} 2 \mathrm{C}$ & $119.8(15)$ & $\mathrm{C} 10-\mathrm{C} 11-\mathrm{C} 12$ & $121.2(2)$ \\
\hline $\mathrm{O} 1-\mathrm{C} 1-\mathrm{C} 2$ & $112.2(2)$ & $\mathrm{C} 10-\mathrm{C} 11-\mathrm{H} 11$ & 119.4 \\
\hline $\mathrm{O} 1-\mathrm{C} 1-\mathrm{H} 1 \mathrm{~A}$ & 109.2 & $\mathrm{C} 12-\mathrm{C} 11-\mathrm{H} 11$ & 119.4 \\
\hline $\mathrm{C} 2-\mathrm{C} 1-\mathrm{H} 1 \mathrm{~A}$ & 109.2 & $\mathrm{~N} 2-\mathrm{C} 12-\mathrm{C} 11$ & $119.9(2)$ \\
\hline $\mathrm{O} 1-\mathrm{C} 1-\mathrm{H} 1 \mathrm{~B}$ & 109.2 & $\mathrm{~N} 2-\mathrm{C} 12-\mathrm{C} 13$ & $122.2(2)$ \\
\hline $\mathrm{C} 2-\mathrm{C} 1-\mathrm{H} 1 \mathrm{~B}$ & 109.2 & $\mathrm{C} 11-\mathrm{C} 12-\mathrm{C} 13$ & $117.9(2)$ \\
\hline $\mathrm{H} 1 \mathrm{~A}-\mathrm{C} 1-\mathrm{H} 1 \mathrm{~B}$ & 107.9 & $\mathrm{C} 14-\mathrm{C} 13-\mathrm{C} 12$ & $120.7(2)$ \\
\hline $\mathrm{N} 1-\mathrm{C} 2-\mathrm{C} 1$ & $109.9(2)$ & $\mathrm{C} 14-\mathrm{C} 13-\mathrm{H} 13$ & 119.7 \\
\hline $\mathrm{N} 1-\mathrm{C} 2-\mathrm{H} 2 \mathrm{~A}$ & 109.7 & $\mathrm{C} 12-\mathrm{C} 13-\mathrm{H} 13$ & 119.7 \\
\hline $\mathrm{C} 1-\mathrm{C} 2-\mathrm{H} 2 \mathrm{~A}$ & 109.7 & $\mathrm{C} 13-\mathrm{C} 14-\mathrm{C} 9$ & $120.7(2)$ \\
\hline $\mathrm{N} 1-\mathrm{C} 2-\mathrm{H} 2 \mathrm{~B}$ & 109.7 & $\mathrm{C} 13-\mathrm{C} 14-\mathrm{H} 14$ & 119.7 \\
\hline $\mathrm{C} 1-\mathrm{C} 2-\mathrm{H} 2 \mathrm{~B}$ & 109.7 & $\mathrm{C} 9-\mathrm{C} 14-\mathrm{H} 14$ & 119.7 \\
\hline $\mathrm{H} 2 \mathrm{~A}-\mathrm{C} 2-\mathrm{H} 2 \mathrm{~B}$ & 108.2 & $\mathrm{~N} 2-\mathrm{C} 15-\mathrm{C} 16$ & $111.2(2)$ \\
\hline $\mathrm{N} 1-\mathrm{C} 3-\mathrm{C} 4$ & $123.1(2)$ & $\mathrm{N} 2-\mathrm{C} 15-\mathrm{H} 15 \mathrm{~A}$ & 109.4 \\
\hline $\mathrm{N} 1-\mathrm{C} 3-\mathrm{C} 8$ & $119.2(2)$ & $\mathrm{C} 16-\mathrm{C} 15-\mathrm{H} 15 \mathrm{~A}$ & 109.4 \\
\hline $\mathrm{C} 4-\mathrm{C} 3-\mathrm{C} 8$ & $117.7(2)$ & $\mathrm{N} 2-\mathrm{C} 15-\mathrm{H} 15 \mathrm{~B}$ & 109.4 \\
\hline $\mathrm{C} 5-\mathrm{C} 4-\mathrm{C} 3$ & $120.9(2)$ & $\mathrm{C} 16-\mathrm{C} 15-\mathrm{H} 15 \mathrm{~B}$ & 109.4 \\
\hline $\mathrm{C} 5-\mathrm{C} 4-\mathrm{H} 4 \mathrm{~A}$ & 119.6 & $\mathrm{H} 15 \mathrm{~A}-\mathrm{C} 15-\mathrm{H} 15 \mathrm{~B}$ & 108.0 \\
\hline $\mathrm{C} 3-\mathrm{C} 4-\mathrm{H} 4 \mathrm{~A}$ & 119.6 & $\mathrm{O} 4-\mathrm{C} 16-\mathrm{C} 15$ & $113.5(2)$ \\
\hline $\mathrm{C} 4-\mathrm{C} 5-\mathrm{C} 6$ & $120.3(2)$ & $\mathrm{O} 4-\mathrm{C} 16-\mathrm{H} 16 \mathrm{~A}$ & 108.9 \\
\hline $\mathrm{C} 4-\mathrm{C} 5-\mathrm{H} 5$ & 119.8 & $\mathrm{C} 15-\mathrm{C} 16-\mathrm{H} 16 \mathrm{~A}$ & 108.9 \\
\hline $\mathrm{C} 6-\mathrm{C} 5-\mathrm{H} 5$ & 119.8 & $\mathrm{O} 4-\mathrm{C} 16-\mathrm{H} 16 \mathrm{~B}$ & 108.9 \\
\hline $\mathrm{C} 5-\mathrm{C} 6-\mathrm{C} 7$ & $119.4(2)$ & $\mathrm{C} 15-\mathrm{C} 16-\mathrm{H} 16 \mathrm{~B}$ & 108.9 \\
\hline $\mathrm{C} 5-\mathrm{C} 6-\mathrm{S} 1$ & $120.64(18)$ & $\mathrm{H} 16 \mathrm{~A}-\mathrm{C} 16-\mathrm{H} 16 \mathrm{~B}$ & 107.7 \\
\hline $\mathrm{C} 3-\mathrm{N} 1-\mathrm{C} 2-\mathrm{C} 1$ & $-175.9(2)$ & $\mathrm{O} 2-\mathrm{S} 1-\mathrm{C} 9-\mathrm{C} 14$ & $161.83(17)$ \\
\hline $\mathrm{O} 1-\mathrm{C} 1-\mathrm{C} 2-\mathrm{N} 1$ & $60.4(3)$ & $\mathrm{O} 3-\mathrm{S} 1-\mathrm{C} 9-\mathrm{C} 14$ & $33.5(2)$ \\
\hline $\mathrm{C} 2-\mathrm{N} 1-\mathrm{C} 3-\mathrm{C} 4$ & $-3.1(3)$ & $\mathrm{C} 6-\mathrm{S} 1-\mathrm{C} 9-\mathrm{C} 14$ & $-81.1(2)$ \\
\hline $\mathrm{C} 2-\mathrm{N} 1-\mathrm{C} 3-\mathrm{C} 8$ & $176.8(2)$ & $\mathrm{O} 2-\mathrm{S} 1-\mathrm{C} 9-\mathrm{C} 10$ & $-20.3(2)$ \\
\hline $\mathrm{N} 1-\mathrm{C} 3-\mathrm{C} 4-\mathrm{C} 5$ & $-179.1(2)$ & $\mathrm{O} 3-\mathrm{S} 1-\mathrm{C} 9-\mathrm{C} 10$ & $-148.70(18)$ \\
\hline $\mathrm{C} 8-\mathrm{C} 3-\mathrm{C} 4-\mathrm{C} 5$ & $1.0(3)$ & $\mathrm{C} 6-\mathrm{S} 1-\mathrm{C} 9-\mathrm{C} 10$ & $96.8(2)$ \\
\hline $\mathrm{C} 3-\mathrm{C} 4-\mathrm{C} 5-\mathrm{C} 6$ & $-0.2(3)$ & $\mathrm{C} 14-\mathrm{C} 9-\mathrm{C} 10-\mathrm{C} 11$ & $-1.3(3)$ \\
\hline $\mathrm{C} 4-\mathrm{C} 5-\mathrm{C} 6-\mathrm{C} 7$ & $-0.4(3)$ & $\mathrm{S} 1-\mathrm{C} 9-\mathrm{C} 10-\mathrm{C} 11$ & $-179.14(18)$ \\
\hline $\mathrm{C} 4-\mathrm{C} 5-\mathrm{C} 6-\mathrm{S} 1$ & 177.07 (17) & $\mathrm{C} 9-\mathrm{C} 10-\mathrm{C} 11-\mathrm{C} 12$ & $0.9(3)$ \\
\hline $\mathrm{O} 2-\mathrm{S} 1-\mathrm{C} 6-\mathrm{C} 5$ & $37.3(2)$ & $\mathrm{C} 15-\mathrm{N} 2-\mathrm{C} 12-\mathrm{C} 11$ & $177.4(2)$ \\
\hline $\mathrm{O} 3-\mathrm{S} 1-\mathrm{C} 6-\mathrm{C} 5$ & $166.02(17)$ & $\mathrm{C} 15-\mathrm{N} 2-\mathrm{C} 12-\mathrm{C} 13$ & $-2.2(3)$ \\
\hline $\mathrm{C} 9-\mathrm{S} 1-\mathrm{C} 6-\mathrm{C} 5$ & $-79.2(2)$ & $\mathrm{C} 10-\mathrm{C} 11-\mathrm{C} 12-\mathrm{N} 2$ & $-179.1(2)$ \\
\hline $\mathrm{O} 2-\mathrm{S} 1-\mathrm{C} 6-\mathrm{C} 7$ & $-145.26(17)$ & $\mathrm{C} 10-\mathrm{C} 11-\mathrm{C} 12-\mathrm{C} 13$ & $0.5(3)$ \\
\hline
\end{tabular}




$\begin{array}{llll}\mathrm{O} 3-\mathrm{S} 1-\mathrm{C} 6-\mathrm{C} 7 & -16.6(2) & \mathrm{N} 2-\mathrm{C} 12-\mathrm{C} 13-\mathrm{C} 14 & 178.0(2) \\ \mathrm{C} 9-\mathrm{S} 1-\mathrm{C} 6-\mathrm{C} 7 & 98.25(19) & \mathrm{C} 11-\mathrm{C} 12-\mathrm{C} 13-\mathrm{C} 14 & -1.6(3) \\ \mathrm{C} 5-\mathrm{C} 6-\mathrm{C} 7-\mathrm{C} 8 & 0.0(3) & \mathrm{C} 12-\mathrm{C} 13-\mathrm{C} 14-\mathrm{C} 9 & 1.2(3) \\ \mathrm{S} 1-\mathrm{C} 6-\mathrm{C} 7-\mathrm{C} 8 & -177.44(17) & \mathrm{C} 10-\mathrm{C} 9-\mathrm{C} 14-\mathrm{C} 13 & 0.3(3) \\ \mathrm{C} 6-\mathrm{C} 7-\mathrm{C} 8-\mathrm{C} 3 & 0.9(3) & \mathrm{S} 1-\mathrm{C} 9-\mathrm{C} 14-\mathrm{C} 13 & 178.15(17) \\ \mathrm{N} 1-\mathrm{C} 3-\mathrm{C} 8-\mathrm{C} 7 & 178.8(2) & \mathrm{C} 12-\mathrm{N} 2-\mathrm{C} 15-\mathrm{C} 16 & -166.6(2) \\ \mathrm{C} 4-\mathrm{C} 3-\mathrm{C} 8-\mathrm{C} 7 & -1.4(3) & \mathrm{N} 2-\mathrm{C} 15-\mathrm{C} 16-\mathrm{O} 4 & 61.0(3)\end{array}$

Hydrogen-bond geometry $\left(A,{ }^{\circ}\right)$

\begin{tabular}{lllll}
\hline$D-\mathrm{H} \cdots A$ & $D-\mathrm{H}$ & $\mathrm{H} \cdots A$ & $D \cdots A$ & $D-\mathrm{H} \cdots A$ \\
\hline $\mathrm{C} 14-\mathrm{H} 14 \cdots \mathrm{O} 3$ & 0.93 & 2.59 & $2.919(3)$ & 101 \\
$\mathrm{C} 10-\mathrm{H} 10 \cdots \mathrm{O} 2$ & 0.93 & 2.57 & $2.915(3)$ & 102 \\
$\mathrm{C} 7-\mathrm{H} 7 \cdots \mathrm{O} 3$ & 0.93 & 2.47 & $2.854(3)$ & 105 \\
$\mathrm{O} 1-\mathrm{H} 1 \cdots \mathrm{O} 3^{\mathrm{i}}$ & 0.82 & 1.87 & $2.683(3)$ & 175 \\
$\mathrm{O} 1-\mathrm{H} 1 \cdots \mathrm{S} 1^{\mathrm{i}}$ & 0.82 & 2.88 & $3.638(2)$ & 154 \\
$\mathrm{O} 4-\mathrm{H} 4 \cdots 2^{\mathrm{ii}}$ & 0.82 & 2.13 & $2.945(3)$ & 177 \\
$\mathrm{~N} 1-\mathrm{H} 1 C \cdots \mathrm{O} 4^{\mathrm{iii}}$ & $0.89(1)$ & $2.19(1)$ & $3.066(3)$ & $169(2)$ \\
$\mathrm{N} 2-\mathrm{H} 2 C \cdots \mathrm{O} 1^{\text {iv }}$ & $0.90(1)$ & $2.03(1)$ & $2.917(3)$ & $172(2)$ \\
$\mathrm{C} 10-\mathrm{H} 10 \cdots C g 2^{\mathrm{v}}$ & 0.93 & 2.97 & $3.762(4)$ & 144
\end{tabular}

Symmetry codes: (i) $-x, y+1,-z+1 / 2$; (ii) $-x+1 / 2, y-1 / 2,-z+1 / 2$; (iii) $x-1 / 2, y+1 / 2, z$; (iv) $x+1 / 2, y-1 / 2, z$; (v) $-x+1 / 2, y+1 / 2,-z+1 / 2$. 